\title{
A Novel Approach Based on MEMS-Gyro's Data Deep Coupling for Determining the Centroid of Star Spot
}

\author{
Xing Fei, Chen Nan, You Zheng, and Sun Ting \\ State Key Laboratory of Precision Measurement Technology and Instruments, \\ Department of Precision Instruments and Mechanology, Tsinghua University, \\ Beijing 100084, China \\ Correspondence should be addressed to Xing Fei, xingfei@tsinghua.edu.cn
}

Received 28 May 2012; Accepted 3 August 2012

Academic Editor: Mohammad Younis

Copyright (c) 2012 Xing Fei et al. This is an open access article distributed under the Creative Commons Attribution License, which permits unrestricted use, distribution, and reproduction in any medium, provided the original work is properly cited.

The traditional approach of star tracker for determining the centroid of spot requires enough energy and good shape, so a relatively long exposure time and stable three-axis state become necessary conditions to maintain high accuracy, these limit its update rate and dynamic performance. In view of these issues, this paper presents an approach for determining the centroid of star spot which based on MEMS-Gyro's data deep coupling, it achieves the deep fusion of the data of star tracker and MEMS-Gyro at star map level through the introduction of EKF. The trajectory predicted by using the angular velocity of three axes can be used to set the extraction window, this enhances the dynamic performance because of the accurate extraction when the satellite has angular speed. The optimal estimations of the centroid position and the drift in the output signal of MEMS-Gyro through this approach reduce the influence of noise of the detector on accuracy of the traditional approach for determining the centroid and effectively correct the output signal of MEMS-Gyro. At the end of this paper, feasibility of this approach is verified by simulation.

\section{Introduction}

In recent years, along with the rapid development of aerospace industry, missions especially to high-precision positioning for imaging system, such as survey, resource, and astronomical satellite, require attitude determination system of higher accuracy. In the attitude determination system, only star tracker can meet the accuracy requirement of these satellites. 


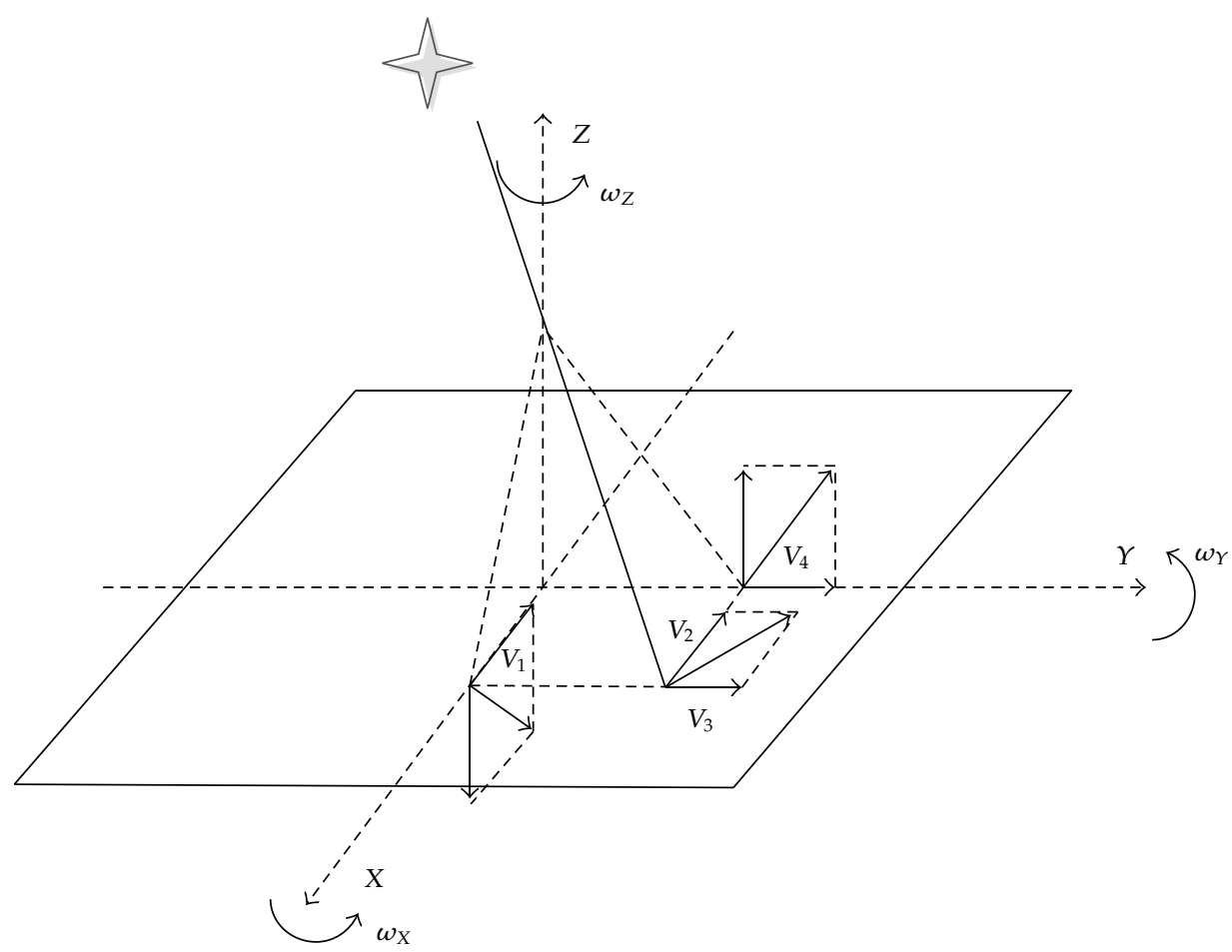

Figure 1: The decomposition of line velocity equivalent with the three-axis angular velocity.

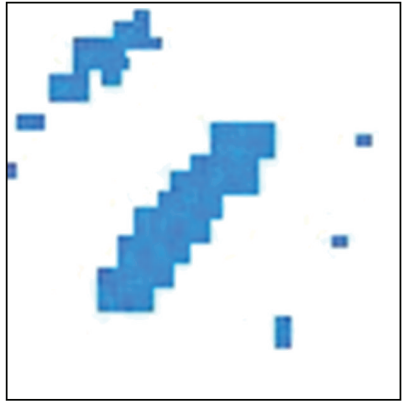

(a) Star spot

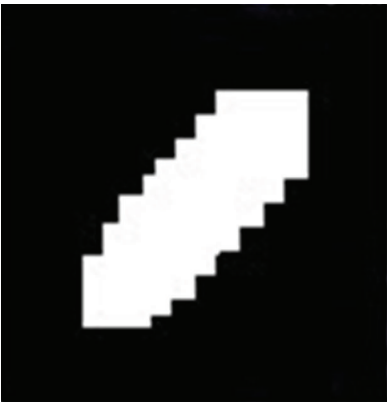

(b) Extraction window

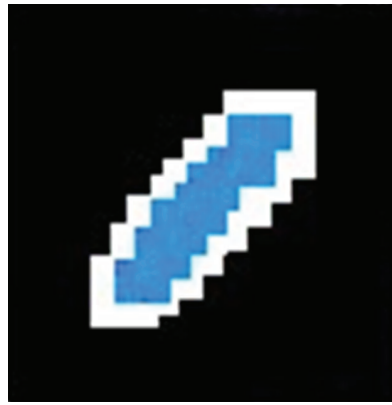

(c) Extract spot

Figure 2: The set of extraction window and the extraction of the spot when the satellite move with a certain angular velocity.

Star tracker is a high-precision optical sensor for determining the attitude of satellite which uses the star map imaging on optical detector. The precision of star tracker directly related to the accuracy of determination of the spot centroid. The traditional approach for determination of the spot centroid requires enough energy and good shape [1]. It can get a subpixel precision of the determination of spot centroid through using hyperacuity technique [2]. So, in order to maintain the accuracy of the spot centroid, it requires a relatively long exposure time and stable three-axis state, these limit its update rate and dynamic performance [3]. The high demand of star tracker to stable state of the satellite makes star tracker can only 


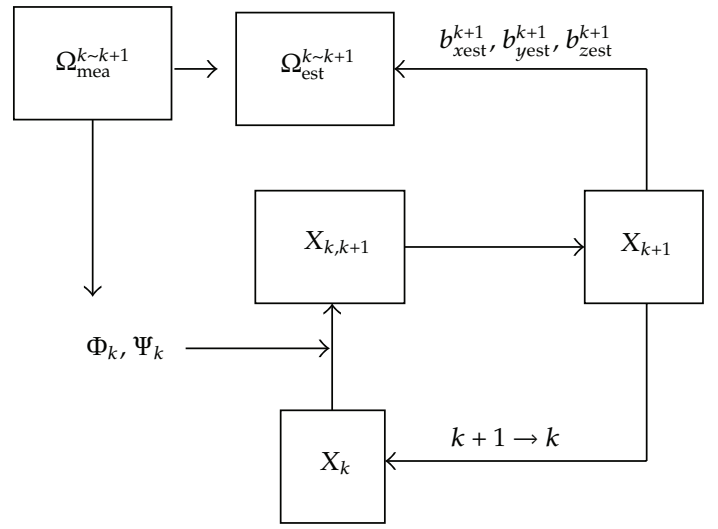

Figure 3: The flow chart of the approach for determination of the position of centroid of the spot based on MEMS-Gyro when the star map has not been updated.

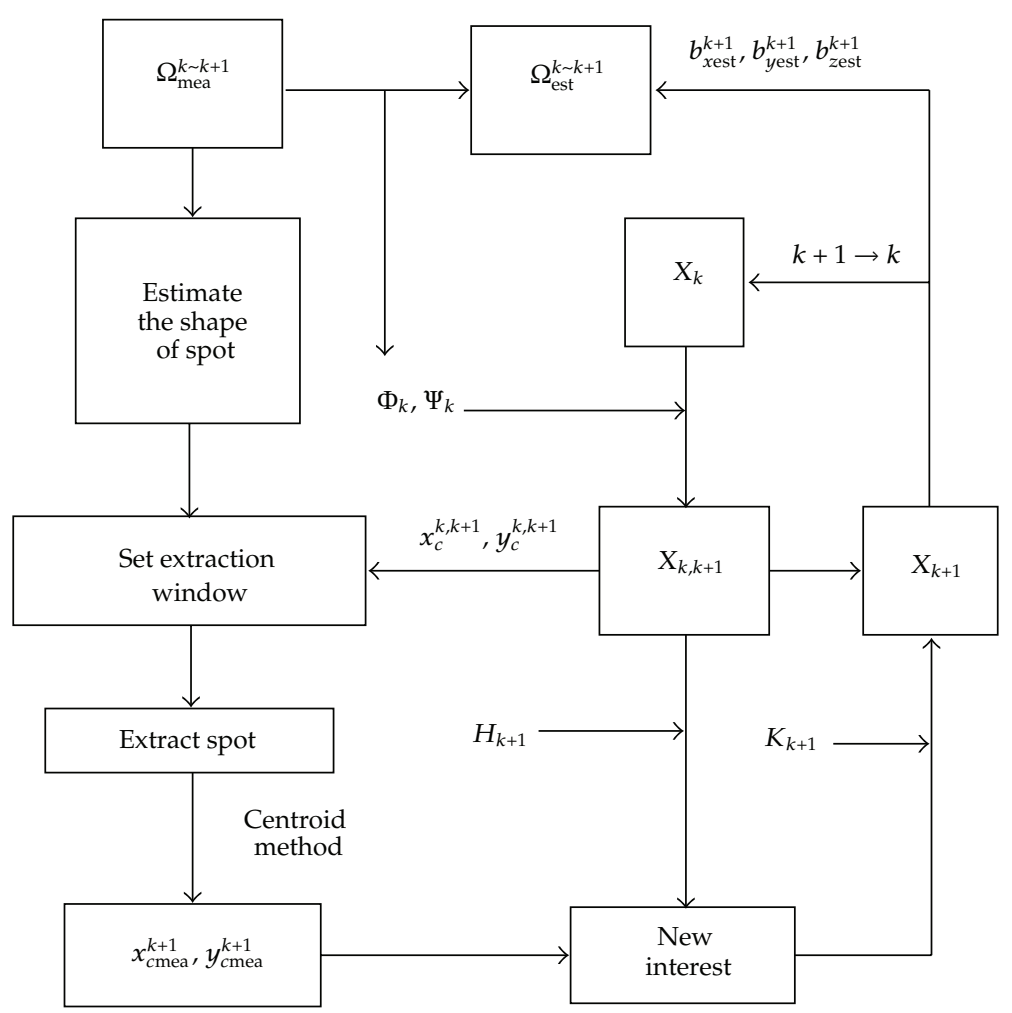

Figure 4: The flow chart of the approach for determination of the position of centroid of the spot based on MEMS-Gyro when the star map has been updated.

maintain precision in condition close to three-axis stabilized state. When the satellite has large angular speed, it can only determine attitude by the lower-precision sensor, the failure of star tracker influences the completion of the missions [4-6].

The spot will be elongated when the satellite has large angular speed, and the larger distribution area of energy will lead to reduction of energy on each pixel when the noise will 

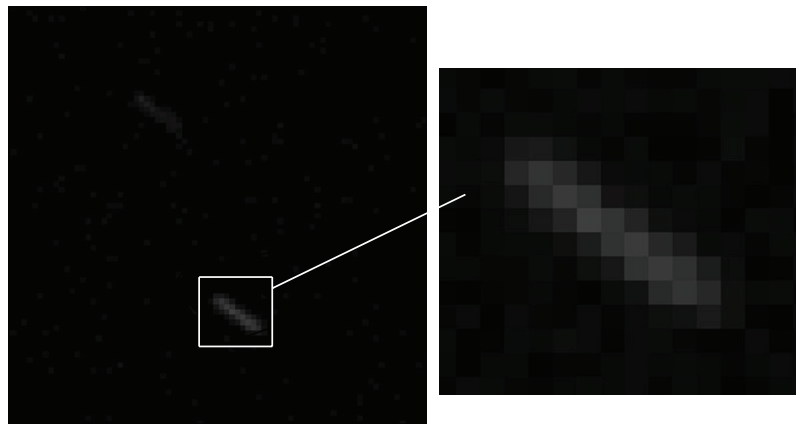

Figure 5: The simulation result with noise of the star map captured when the satellite is moving.

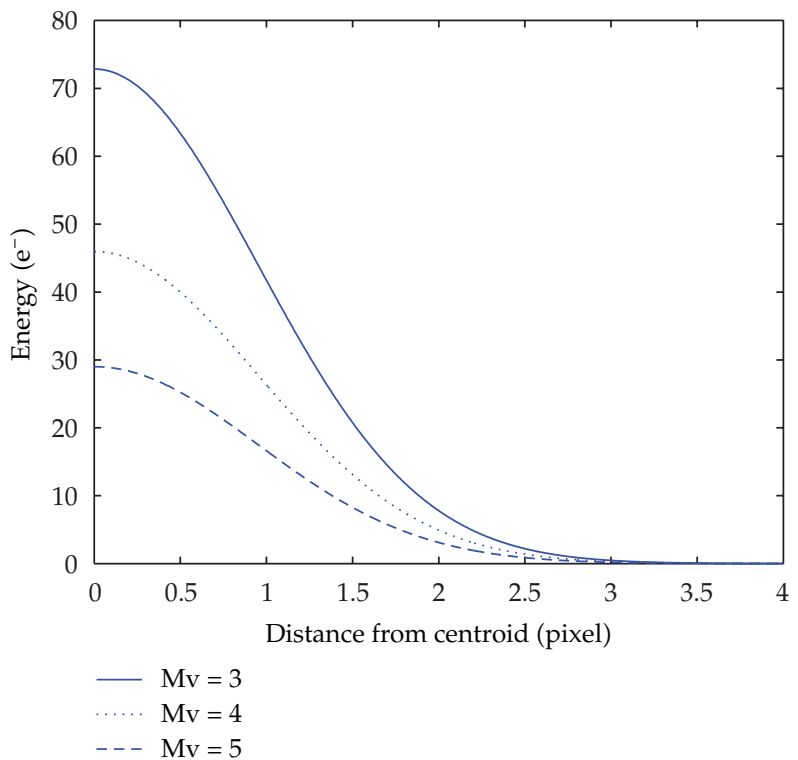

Figure 6: The curve of energy changed by the change of distance from the spot centroid.

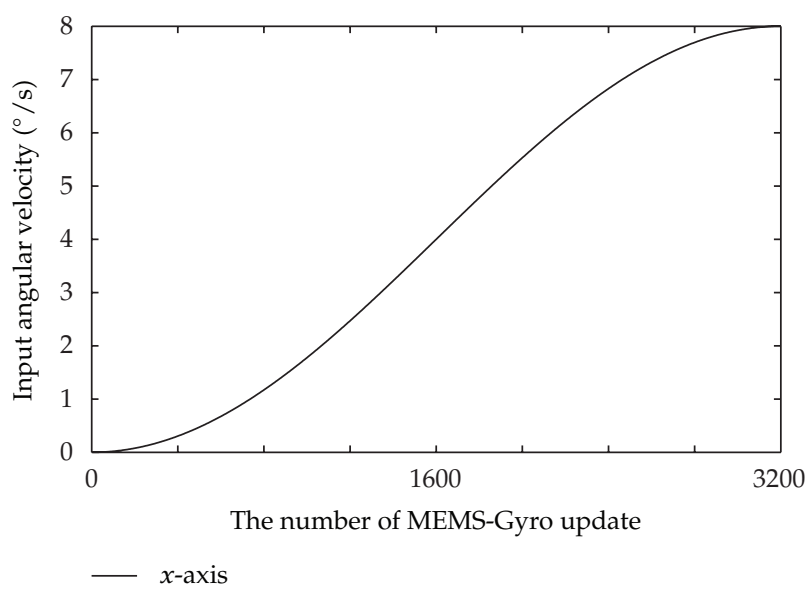

Figure 7: The input angular velocity in Simulation 1. 


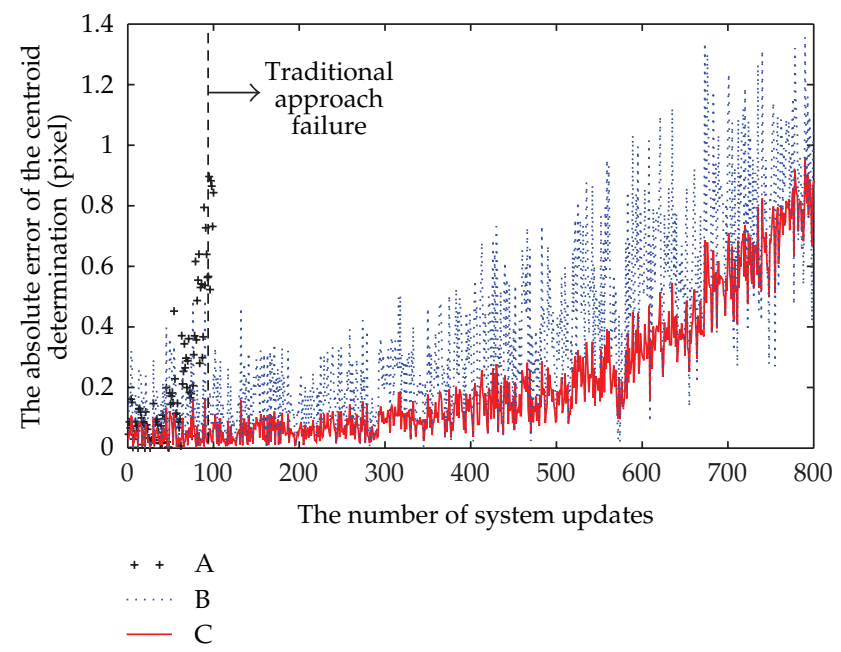

Figure 8: The error of the position of spot centroid (A: the error of the position of the spot centroid using the traditional centroid method, B: the error of the position of spot centroid using the centroid method after the spot extraction based on MEMS-Gyro, and C: the estimation error of the position of the spot centroid using the approach based on MEMS-Gyro's data deep coupling).

not be reduced, so the lower SNR causes a great impact on the star tracker. First, it is more difficult to extract the spot. The reduction of the SNR makes it more difficult to determine the position of the spot, the elongated spot and mutual interference between the spot which caused by it making the determination of area of spot extraction more difficult. Second, it is more difficult to determine the position of spot centroid [7]. To determine the centroid of spot accurately, it needs ideal energy distribution. But, the lower SNR makes the influence of noise greater and then affects the accuracy of the determination of the position of centroid. For the above reasons, star tracker will not work because of inability to spot extraction and centroid determination when the satellite has larger angular speed. These limit the accuracy and dynamic performance of star tracker.

In addition to star tracker, there are many other sensors for attitude determination. MEMS-Gyro which is based on the MEMS technology had successful applications in the aerospace industry, it has advantages of high precision, low power, and small volume [8]. There are many MEMS-Gyros developed by a number of agencies reach the standard of the application of aerospace grade and have experienced in-orbit test, some of them have been quite associated with the optical Gyro. Presently, institutions of high levels of production of MEMS-Gyro are mainly a few such as Draper and JPL [9-11].

To improve the renew rate and dynamic performance of star tracker, some agencies began to research the integrated approach of MEMS-Gyro, such as the ISC developed by Draper and the HYDRA $[12,13]$. The new approach presented in this paper to determine the position of spot centroid is based on MEMS-Gyro's data deeply coupled, this approach achieves the deep fusion of star tracker and MEMS-Gyro at star map level through the introduction of EKF. By applying this approach, the problems caused by lower SNR of star tracker can get effective solution. 


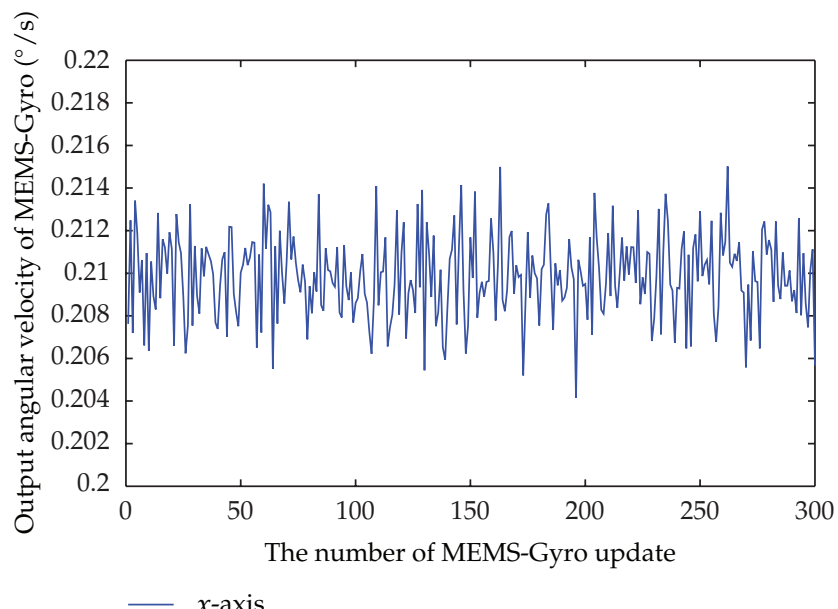

(a)

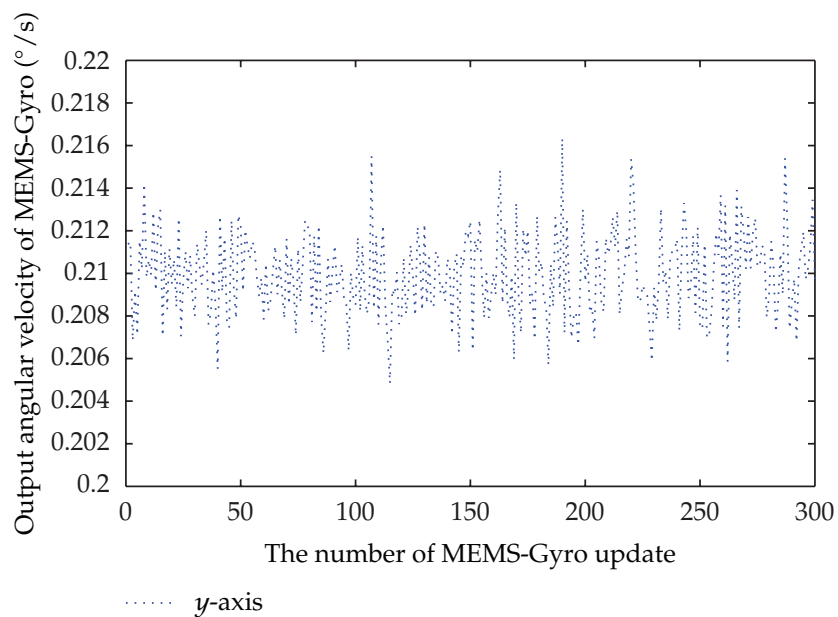

(b)

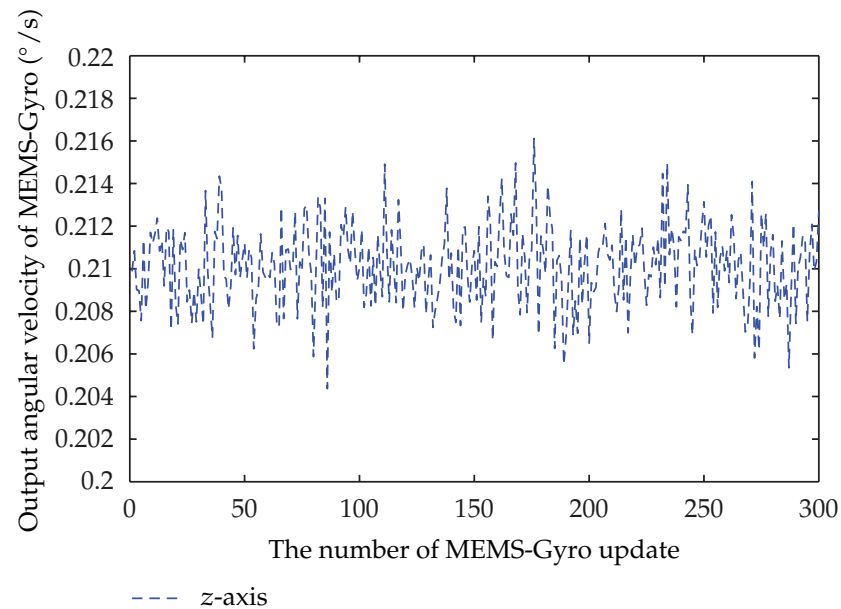

(c)

Figure 9: The measured velocity of three angular. 


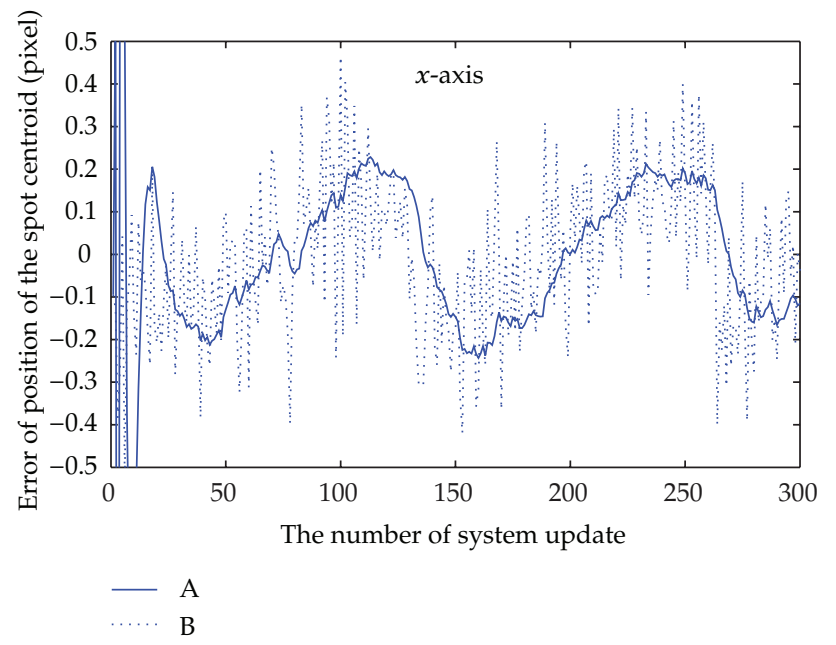

(a)

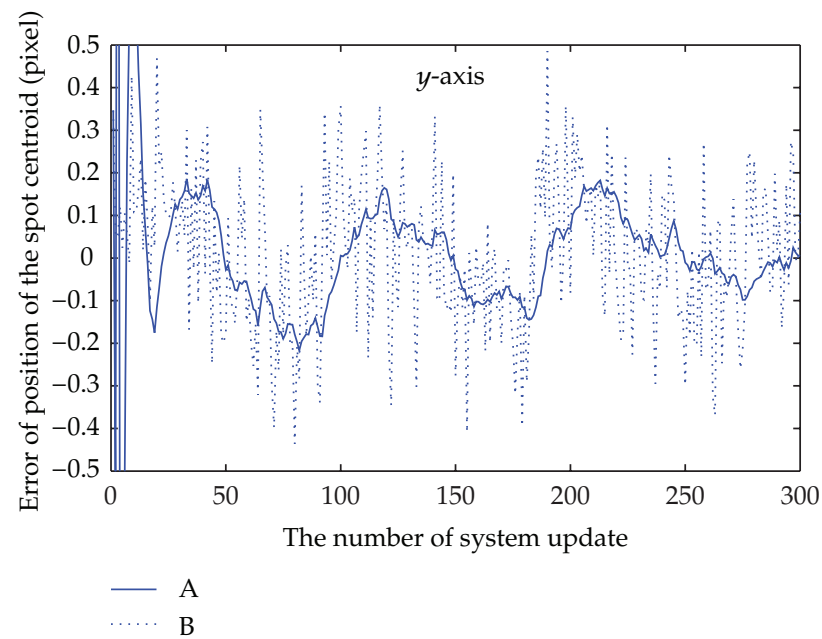

(b)

Figure 10: The error of the position of spot centroid (A: the estimation error of the position of the spot centroid using the approach based on MEMS-Gyro's data deep coupling, B: the error of the position of spot centroid using the centroid method after the spot extraction based on MEMS-Gyro).

\section{Star Spot Extraction Based on MEMS-Gyro}

It is generally considered that the star is at infinity, so the starlight which captured by star tracker is parallel light. When the satellite moves in space, the change of attitude associates with three-axis angular velocity, the position of spot centroid on detector will transform accompanied by the change of attitude. The angular velocity can be equivalent to the line speed on the detector plane. If MEMS-Gyro is integrated into the star tracker, the three-axis angular velocity measured by MEMS-Gyro can be used to calculate the line velocity and direction of the centroid on the detector plane [14]. Then, it can use the position and shape of the spot next time which can be estimated by the motion of the centroid to set the extraction 


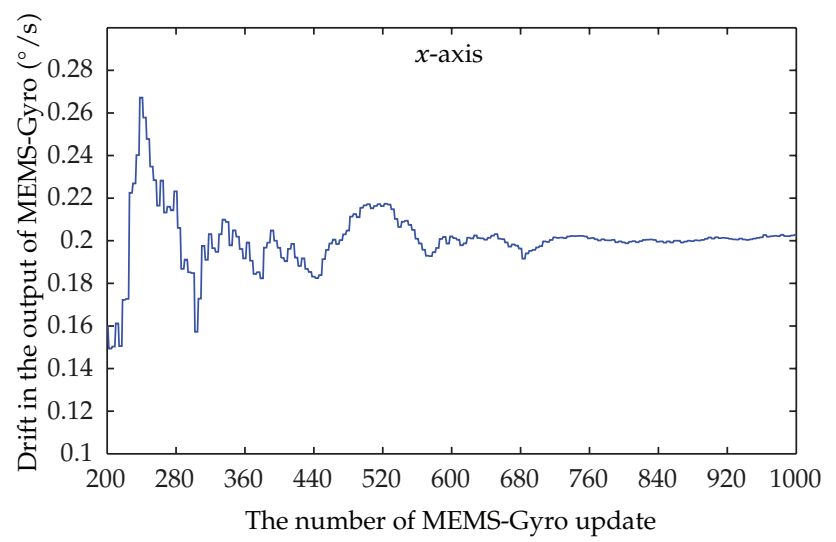

(a)

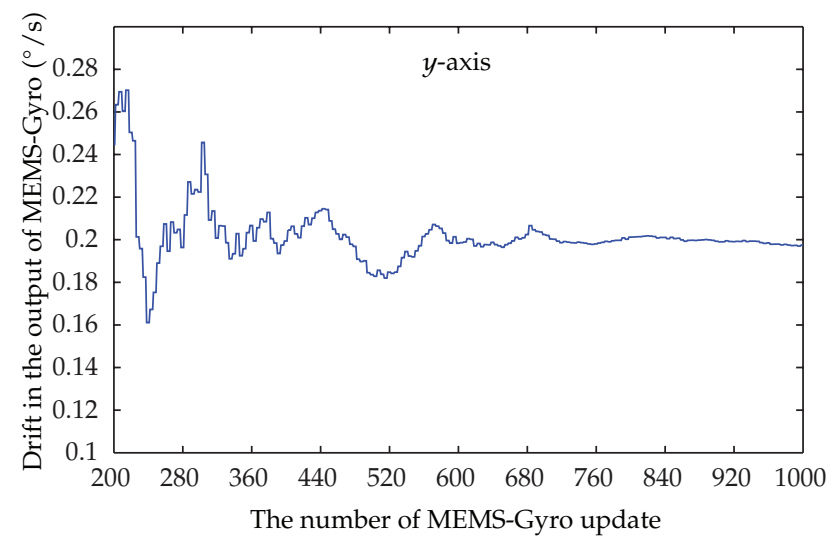

(b)

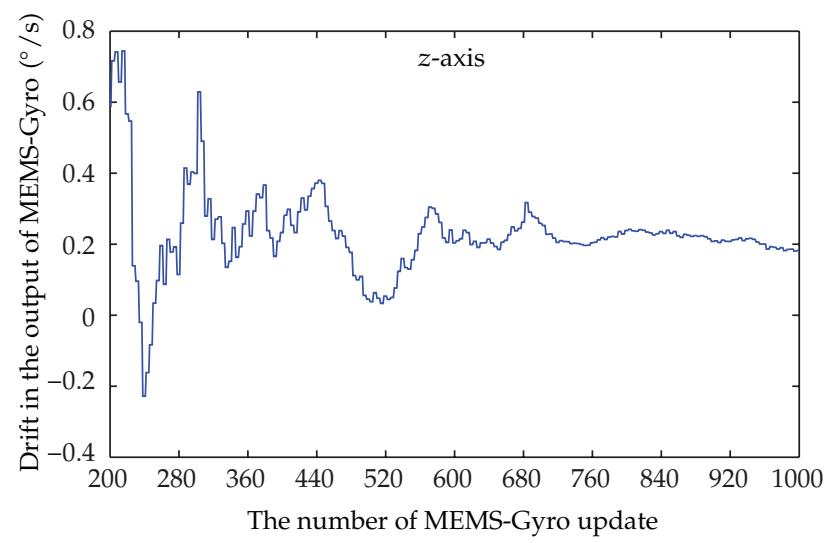

(c)

Figure 11: The estimated drift in the output data of MEMS-Gyro. 


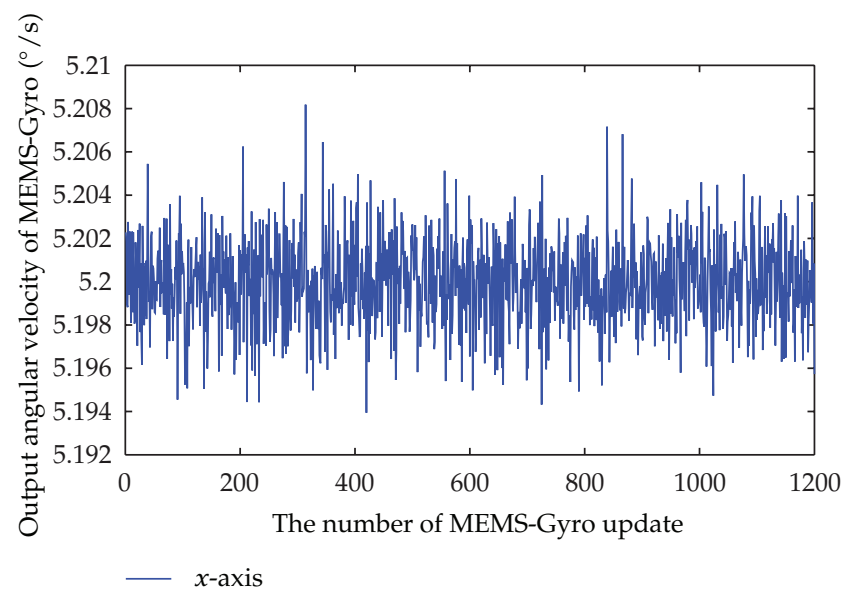

(a)

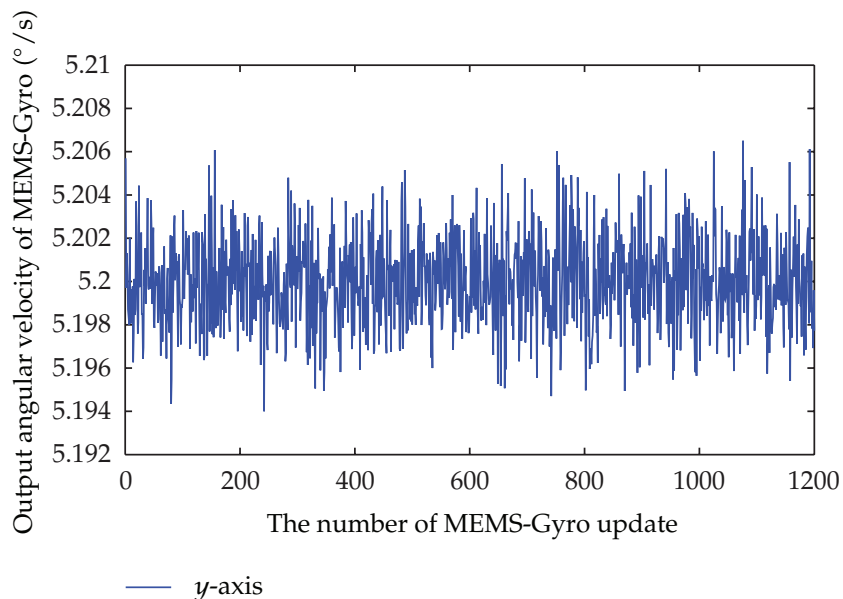

(b)

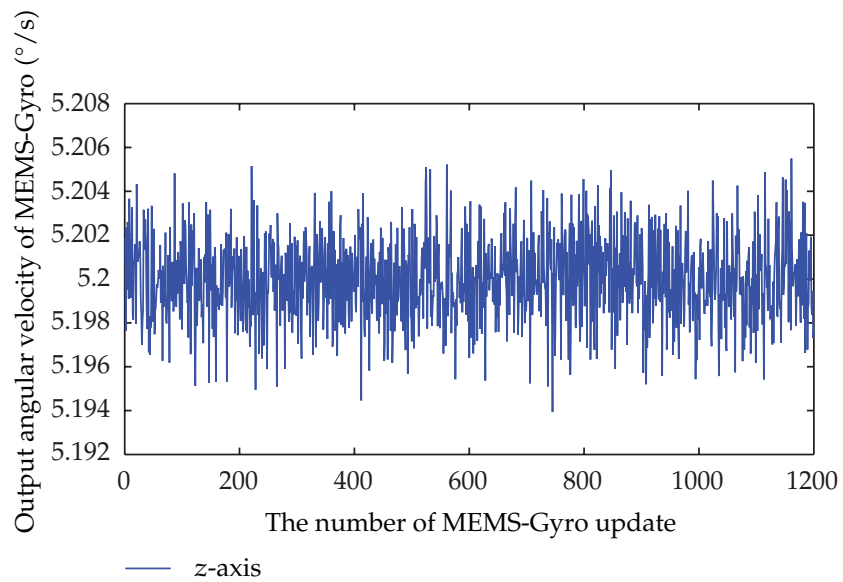

(c)

Figure 12: The measured velocity of three angular. 

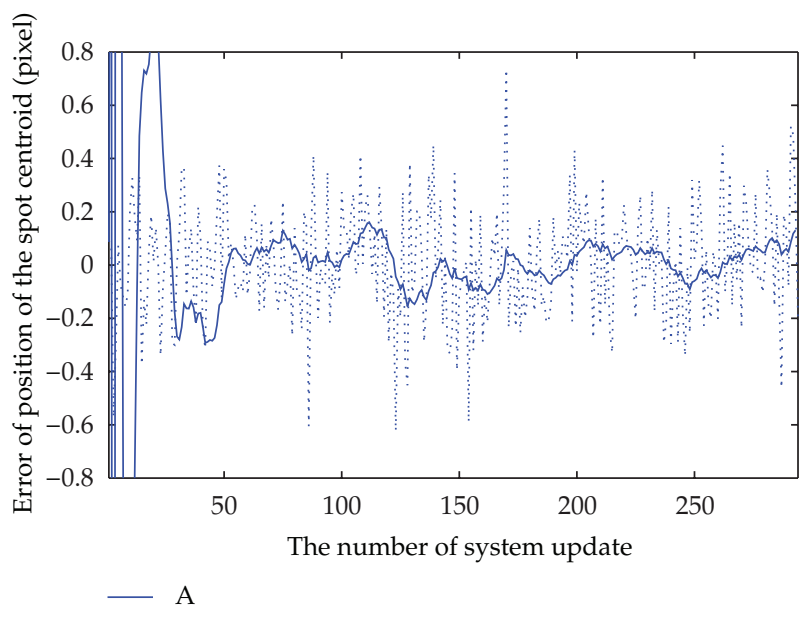

(a)

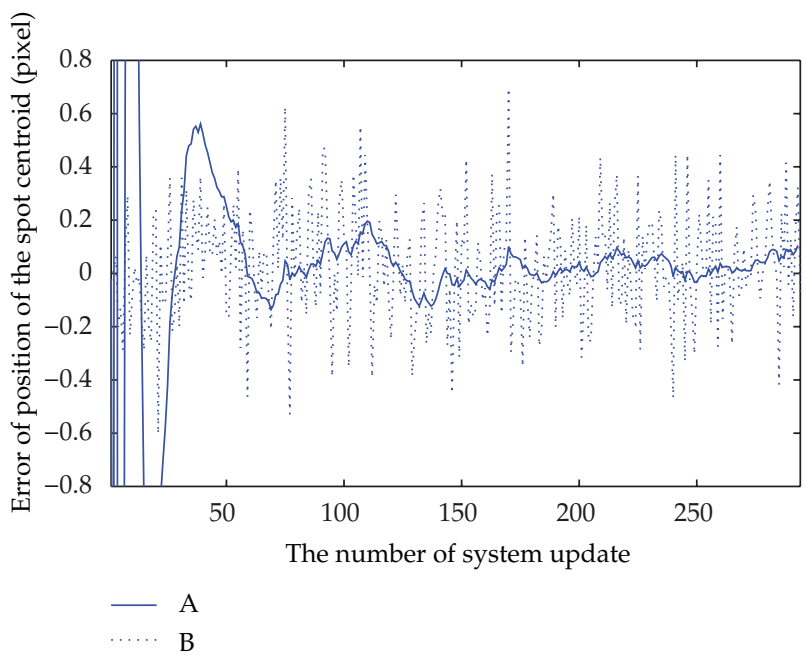

(b)

Figure 13: The error of the position of spot centroid (A: the estimation error of the position of the spot centroid using the approach based on MEMS-Gyro's data deep coupling, B: the error of the position of spot centroid using the centroid method after the spot extraction based on MEMS-Gyro).

window, so the condition that other spot gets into the window could be avoided. It ensures the purity of the energy in the extraction window.

The velocity on the detector plane which is equivalent by three-axis angular velocity is shown in Figure 1. The line velocity equivalent to detector by angular velocity of $x$-axis is $v_{4}$ along the $y$-axis; the line velocity equivalent to detector by angular velocity of $y$-axis is $v_{1}$ along the $x$-axis; the effect of the angular velocity of $Z$-axis on detector plane can be 


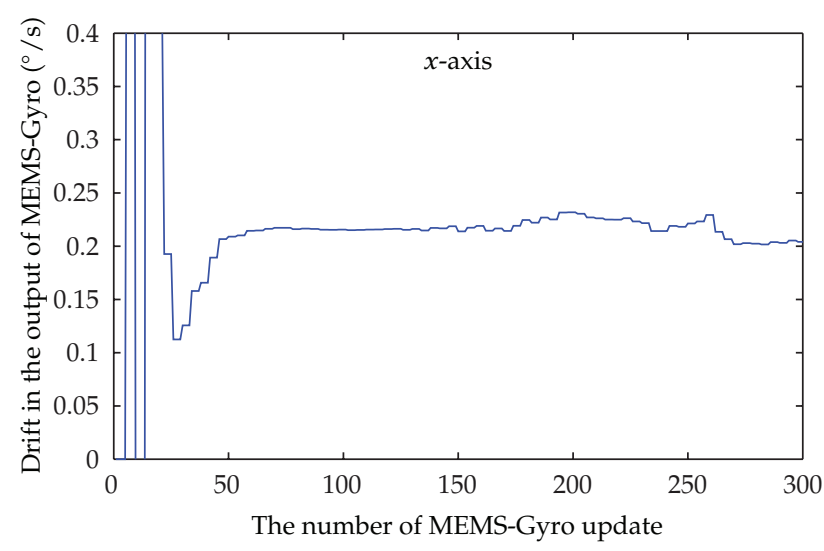

(a)

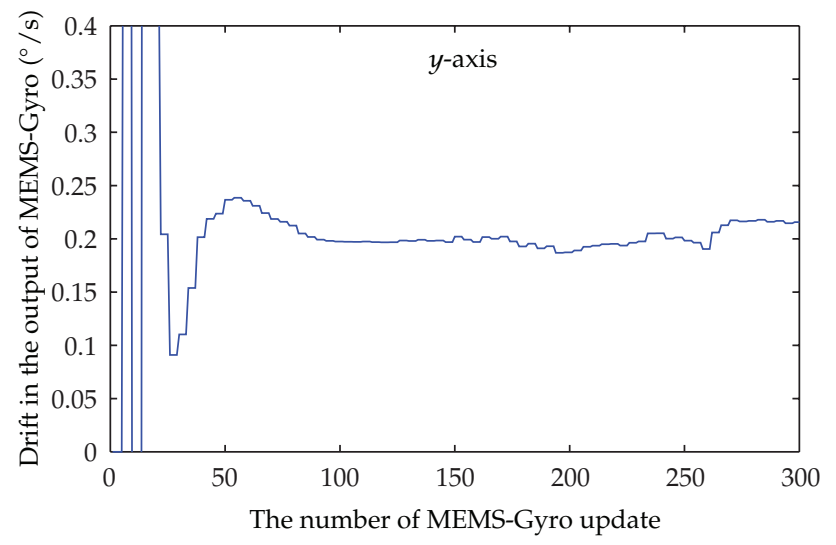

(b)

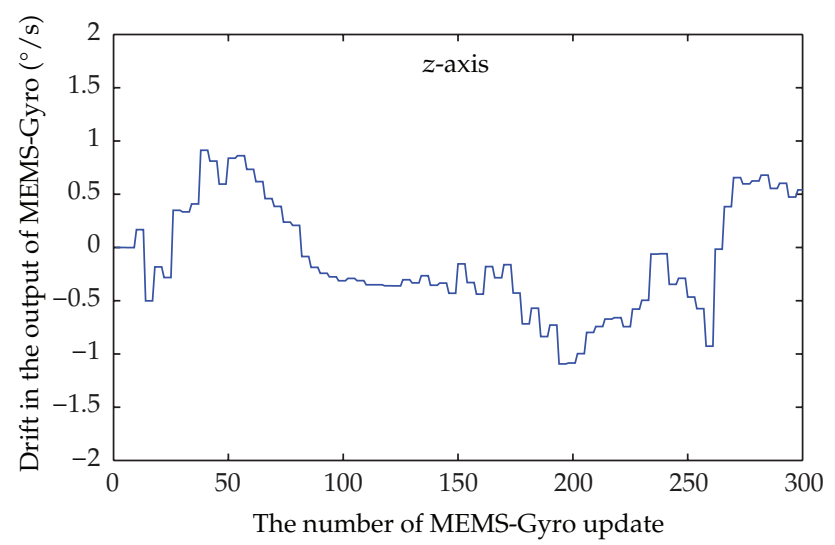

(c)

Figure 14: The estimated drift in the output data of MEMS-Gyro. 


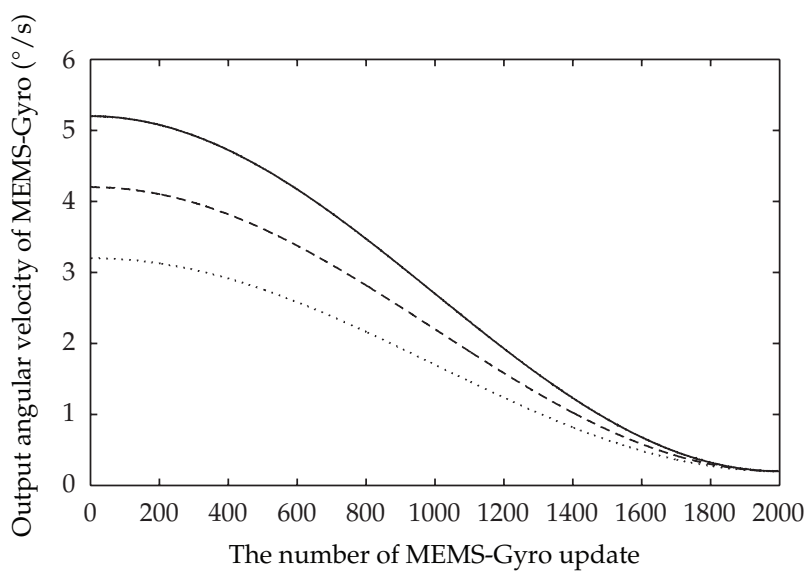

Figure 15: The measured velocity of three angular.

decomposed into $v_{3}$ along the $y$-axis and $v_{2}$ along the $x$-axis. So, the motion of the centroid on the detector plane can be described by the line velocity along $x$-axis and $y$-axis:

$$
\begin{gathered}
V_{x}=-\omega_{z} \times y_{c}-\omega_{y} \times F, \\
V_{y}=\omega_{z} \times x_{c}+\omega_{x} \times F,
\end{gathered}
$$

where $F$ is focal length of the star tracker, $\left(x_{c}, y_{c}\right)$ is coordinate of the centroid of spot.

Assume that $\left(x_{c}^{t_{k}}, y_{c}^{t_{k}}\right)$ is the coordinate of centroid at $t_{k}$, the three-axis angular velocity measured by MEMS-Gyro are $\omega_{x}^{t_{k}}, \omega_{y}^{t_{k}}$, and $\omega_{z}^{t_{k}}$. According to (2.1a) and (2.1b), $V_{x}^{t_{k}}$ and $V_{y}^{t_{k}}$ which are the line velocity of centroid of the spot can be calculated. So, $\left(x_{c}^{t_{k+1}}, y_{c}^{t_{k+1}}\right)$ which is the coordinate of the centroid at $t_{k+1}$ can be estimated as follows:

$$
\begin{aligned}
& x_{c}^{t_{k+1}}=x_{c}^{t_{k}}+\int_{t_{k}}^{t_{k+1}} V_{x}^{t} d t \\
& y_{c}^{t_{k+1}}=y_{c}^{t_{k}}+\int_{t_{k}}^{t_{k+1}} V_{y}^{t} d t .
\end{aligned}
$$

When $\left(t_{k+1}-t_{k}\right)$ is very small, $(2.2 \mathrm{a})$ and $(2.2 \mathrm{~b})$ can be simplified:

$$
\begin{aligned}
& x_{c}^{t_{k+1}} \approx x_{c}^{t_{k}}+V_{x}^{t_{k}} \times\left(t_{k+1}-t_{k}\right), \\
& y_{c}^{t_{k+1}} \approx y_{c}^{t_{k}}+V_{y}^{t_{k}} \times\left(t_{k+1}-t_{k}\right) .
\end{aligned}
$$

After a single exposure, star tracker can forecast the position of centroid of the spot in the image generated in next exposure using (2.3a) and (2.3b). At the same time, the three-axis 

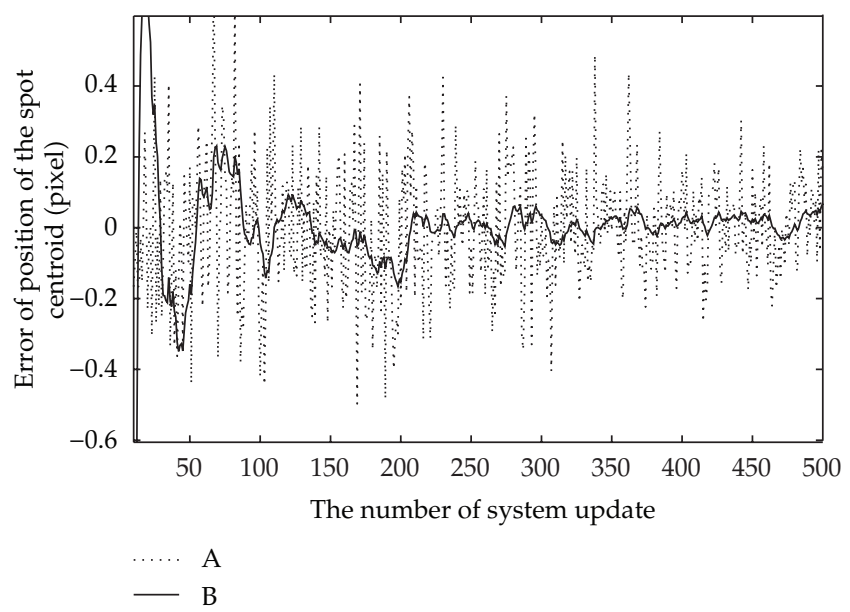

(a)

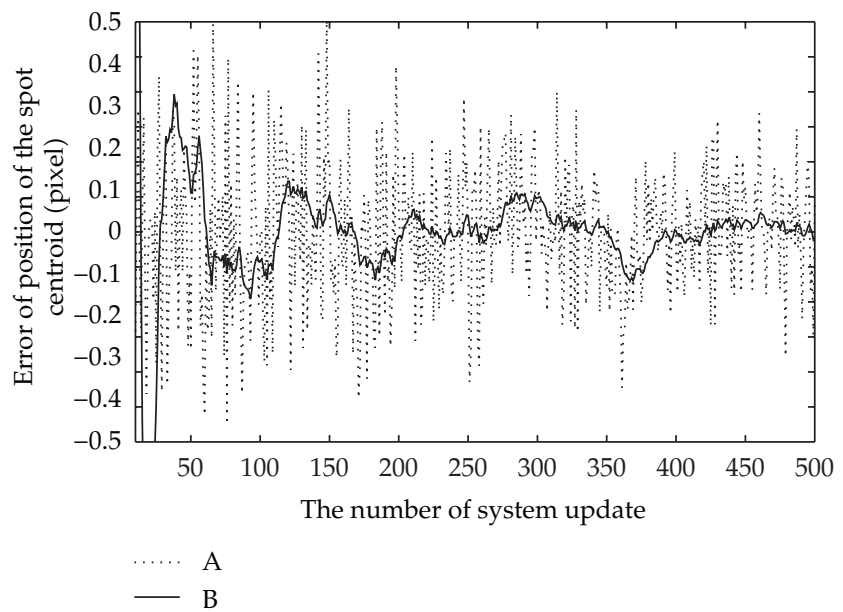

(b)

Figure 16: The error of the position of spot centroid (A: the estimation error of the position of the spot centroid using the approach based on MEMS-Gyro's data deep coupling, B: the error of the position of spot centroid using the centroid method after the spot extraction based on MEMS-Gyro).

angular velocity measured in the time period of next exposure can be used to ensure the shape of spot in next image. Then, star tracker can set extraction window using these information to extract the spot. The process is shown in Figure 2, it can be seen that the extraction window set according to this method well eliminates pepper noise and interference caused by other spot in the extraction window set according to the traditional method.

\section{Determination of Position of the Centroid of Spot Based on EKF}

The most common method to determine the centroid of spot is centroid method and fitting method, they can get high precision when star was imaged with high quality. The centroid method is of high performance-to-price ratio, it has a small amount of calculation and 


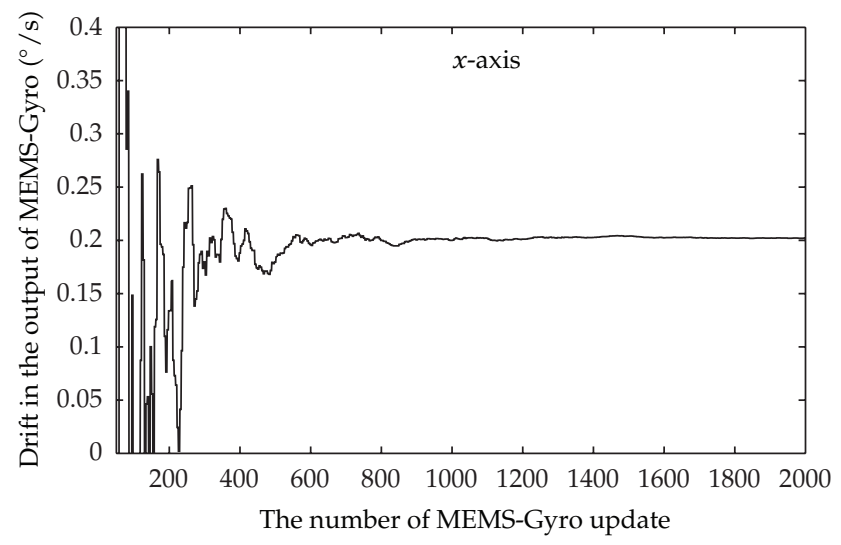

(a)

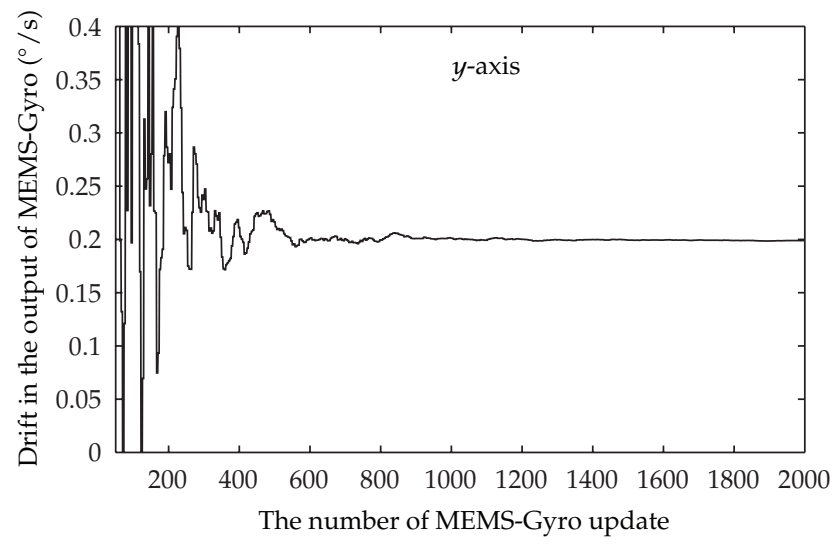

(b)

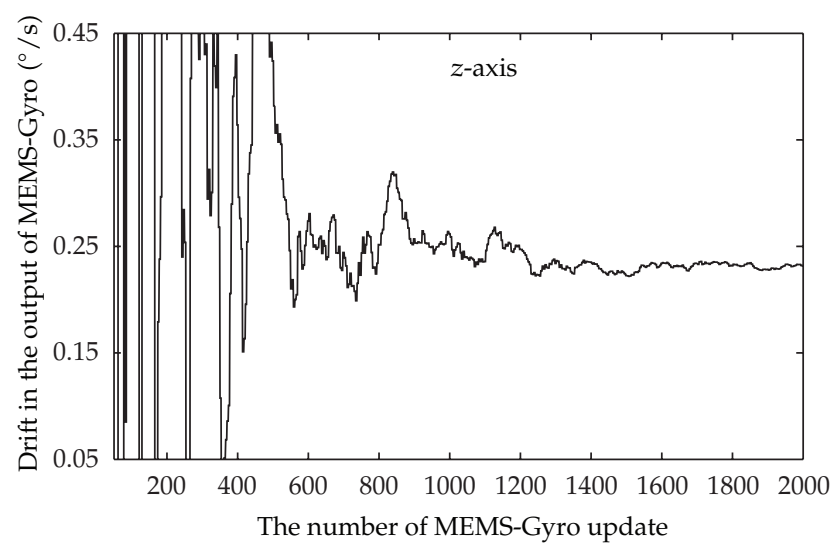

(c)

Figure 17: The estimated drift in the output data of MEMS-Gyro. 
can achieve high precision. But, there exist some errors, the gray scales average effect and quantization error are two main reasons. It makes the position of centroid of the spot determined by the centroid method of certain error [15, 16]. Even so, the centroid method is the most commonly used method because the error is very small when the satellite is in three-axis stabilized state.

As mentioned, when the exposure time is reduced, especially the satellite moves with a large angular speed, the SNR of energy of the detector becomes lower, then the larger effect of noise to the centroid method makes the accuracy of position of centroid determination reduced, further to affect the star tracker.

In view of this problem, it is considered of the form of star tracker based on MEMSGyro, at the same time, the angular velocity output by MEMS-Gyro is used to correct the position determined by the centroid method on the basis of EKF [17-20]. There is the output model of MEMS-Gyro:

$$
\omega_{\text {mea }}=\omega_{\text {real }}+b+\eta_{\omega}
$$

where $\omega_{\text {mea }}$ is the measured value of three-axis angular velocity, $\omega_{\text {real }}$ is the real value of three-axis angular velocity, $b$ is the drift in the output signal of MEMS-Gyro, and $\eta_{\omega}$ is zero mean white Gaussian noise [21, 22]

According to (3.1), when the white Gaussian noise is ignored, (2.1a) and (2.1b) can be presented in the following form:

$$
\begin{gathered}
V_{x}=-\left(\omega_{z \text { mea }}-b_{z}\right) \times y_{c}-\left(\omega_{y \text { mea }}-b_{y}\right) \times F, \\
V_{y}=\left(\omega_{\text {zmea }}-b_{z}\right) \times x_{c}+\left(\omega_{\text {xmea }}-b_{x}\right) \times F .
\end{gathered}
$$

First, the state vector can be set:

$$
X=\left[x_{c}, y_{c}, b_{x}, b_{y}, b_{z}\right]^{T} .
$$

Further, the system state equation can be gotten according to (2.3a), (2.3b) and (3.2a), $(3.2 b)$ :

$$
X_{k+1}=\Phi_{k} X_{k}+\Psi_{k}+w_{k}
$$

where $w_{k}$ is the process noise, it satisfies

$$
E\left[w_{k}\right]=0 .
$$

$\Psi_{k}$ is constant compensation:

$$
\Psi_{k}=\left[\begin{array}{lllll}
-\omega_{y \text { mea }}^{k} & \omega_{x \text { mea }}^{k} & 0 & 0 & 0
\end{array}\right]^{T} \cdot F \cdot \frac{\Delta t}{a},
$$

where $a$ is the length of one pixel on the detector, $\omega_{\text {mea }}^{k}$ is the measured value at $k$ time. 
$\Phi_{k}$ is the state transition matrix, it satisfies

$$
\Phi_{k}=\left[\begin{array}{ccccc}
1 & -\omega_{z \text { mea }}^{k} \Delta t & 0 & \frac{F}{a} \Delta t & y_{\text {cest }}^{k} \Delta t \\
\omega_{z \text { mea }}^{k} \Delta t & 1 & -\frac{F}{a} \Delta t & 0 & -x_{\text {cest }}^{k} \Delta t \\
0 & 0 & 1 & 0 & 0 \\
0 & 0 & 0 & 1 & 0 \\
0 & 0 & 0 & 0 & 1
\end{array}\right],
$$

where $x_{\text {cest }}^{k}, y_{\text {cest }}^{k}$ is the estimated value at $k$ time.

The observation of the system is

$$
Z_{k+1}=H_{k+1} X_{k+1}+v_{k+1}
$$

$x_{c \text { mea }}^{k}, y_{c \text { mea }}^{k}$ is used to present the measured value at $k$ time,

$$
Z_{k+1}=\left[x_{c \text { mea }}^{k+1}, \mathrm{y}_{c \text { mea }}^{k+1}\right]^{T}
$$

$H_{k+1}$ is the observation matrix:

$$
H_{k+1}=\left[\begin{array}{lllll}
1 & 0 & 0 & 0 & 0 \\
0 & 1 & 0 & 0 & 0
\end{array}\right]
$$

$v_{k+1}$ is the observation noise, it satisfies

$$
E\left[v_{k+1}\right]=0 .
$$

$x_{c \text { mea }}^{k}, y_{c \text { mea }}^{k}$ is the position of centroid of the spot calculated by the centroid method.

In accordance with the state equation and observation equation listed above, the position of centroid of the spot can be estimated, the specific processes of the entire method are shown in Figure 3. $\Omega^{k}=\left[\omega_{x}^{k}, \omega_{y}^{k}, \omega_{z}^{k}\right]^{T}$ in the figure.

As shown in Figure 3, when star tracker is exposed and no image updated, the approach works according to the process represented in the flow chart. The next position of the spot centroid is forecasted according to (3.4) using the data of angular velocity output by high update rate MEMS-Gyro, the forecasted position is output directly as the estimate, and then the centroid trajectory which can be used to set the extraction window at next image update will be calculated. The approach works according to the process shown in Figure 4 when star tracker renew the star image. The optimal position of centroid of the spot can be estimated through the correction to the forecasted position by the observation position of the spot centroid using of the update image.

Taking advantage of this approach, the attitude update rate gets large increase. It is of valid estimate to the drift in the output data of MEMS-Gyro, so the attitude error is reduced when the MEMS-Gyro is used alone. At the same time, the approach can help to upgrade the precision of the centroid determination when the precision is at a low level because of 
the change of the exposure time or the motion state, so it ensures the accuracy of the attitude output by the system.

\section{Numerical Simulation}

In order to verify the feasibility of the approach above and evaluate the optimization results, the numerical simulation is carried out. The simulation chooses APS CMOS as the starlight detector, and the relevant parameters are set according to the statistical properties obtained by large number of experiments [23]. The focal length $F$ is set to $100 \mathrm{~mm}$, the lens aperture $D$ is set to $80 \mathrm{~mm}$, the optical transmittance is 0.85 , the exposure time $t_{b}$ is $10 \mathrm{~mm}$, the sampling rate of MEMS-Gyro is $400 \mathrm{~Hz}$, and the drift is $0.02^{\circ} / \mathrm{s}$.

The star map captured when the satellite is moving can be considered to be the superposition of the star map in the static case. So we can simulate the dynamic star map using this method, the simulation result of the dynamic star map is shown in Figure 5 [24].

The setting methods of the size and shape of the extraction window in the simulation are as follows: first, ensure the motion trajectory of the centroid during this exposure time according to the position of the spot centroid in last exposure time and the three-axis angular velocity measured by MEMS-Gyro during this exposure time; second, take a small square window around each pixel along the trajectory, and then all of these small windows stack up to be the extraction window. It can be calculated that the energy will be very small on the point distance from the star spot centroid more than three pixels, as shown in Figure 6 , most energy is concentrated in range of 6 pixel $\times 6$ pixel, so the small window can be set to $6 \times 6$. Simulation 1. The dynamic performance of the novel approach based on MEMS-Gyro's data deep coupling.

This simulation will verify the dynamic performance of the novel approach above. It is against the star which $M v=5$, set the three-axis angular velocity from $\Omega_{\text {real }}=[0,0,0]^{T}$ $(\% / \mathrm{s})$ to $\Omega_{\text {real }}=[8,0,0]^{T}(\% / \mathrm{s})$, the renew rate of the system is $100 \mathrm{~Hz}$, continuous sampling 800 times. The angular velocity of three angular in this simulation is shown in Figure 7. The simulation result is presented in Figure 8, as it can be seen, the traditional approach for centroid determination is failure when the angular velocity larger than $1^{\circ} / \mathrm{s}$ because it cannot extract the spot exactly. Further, when using the method of spot extraction based on MEMSGyro, the traditional approach can continue to work. When the novel approach based on MEMS-Gyro's data deep coupling for determining the centroid of the star spot, the accuracy of the centroid determination is improved obviously.

Simulation 2. The estimate of centroid under the three-axis stabilized state of inertia.

This simulation will verify the high update rate and high precision of the approach above when the satellite is under three-axis stabilized state. It is against the star which $M v=$ 5 , set the three-axis angular velocity $\Omega_{\text {real }}=[0.01,0.01,0.01]^{T}(\% / s)$, the renew rate of the system is $100 \mathrm{~Hz}$, continuous sampling 300 times. The measured velocity of three angular is shown in Figure 9, and the estimation error of the position of the spot centroid is shown in Figure 10. Finally, in Figure 11, the estimated drift in the output data of MEMS-Gyro is shown. In order to show the advantages of this approach better, it is also shown the position of the spot centroid calculated by the centroid method after the spot extraction based on MEMSGyro. It can be seen that the error of the position of the centroid using the approach in this paper is reduced from 0.2 pixel to 0.1 pixel when the SNR is lower and exposure time is only $10 \mathrm{~ms}$. The drift in the output data of MEMS-Gyro has got valid estimates.

Simulation 3. The satellite moves with a large uniform angular velocity. 
This simulation will verify the high update rate, high dynamic performance, and the capability to maintain a certain precision of the approach above when the satellite is moving with large three-axis angular velocity. It is against the star which $M v=5$, set the three-axis angular velocity $\Omega_{\text {real }}=[5,5,5]^{T}(\%$ s), the renew rate of the system is $100 \mathrm{~Hz}$, continuous sampling 300 times. The measured velocity of three angular is shown in Figure 12, and the estimation error of the position of the spot centroid is shown in Figure 13. Finally, in Figure 14, the estimated drift in the output data of MEMS-Gyro is shown. In order to show the advantages of this approach better, it's also shown the position of the spot centroid calculated by the centroid method after the spot extraction based on MEMS-Gyro. It can be seen that the error of the position of the centroid using the approach in this paper is reduced from 0.3 pixel to 0.2 pixel when the SNR is lower and exposure time is only $10 \mathrm{~ms}$. The drift in the output data of MEMS-Gyro has got valid estimates.

Simulation 4 . The satellite moves with a time-variable angular velocity.

This simulation will verify the high update rate, high dynamic performance, and the capability to maintain a certain precision of the approach above when the satellite is moving with time-variable angular velocity. It is against the star which $\mathrm{Mv}=5$, set the three-axis angular velocity reduced from $\Omega_{\text {real }}=[5,5,5]^{T}\left({ }^{\circ} / \mathrm{s}\right)$ to $\Omega_{\text {real }}=[0,0,0]^{T}\left({ }^{\circ} / \mathrm{s}\right)$, the renew rate of the system is $100 \mathrm{~Hz}$, continuous sampling 500 times. The measured velocity of three angular is shown in Figure 15, and the estimation error of the position of the spot centroid is shown in Figure 16. Finally, in Figure 17, the estimated drift in the output data of MEMSGyro is shown. In order to show the advantages of this approach better, it is also shown the position of the spot centroid calculated by the centroid method after the spot extraction based on MEMS-Gyro. It can be seen that the error of the position of the centroid using the approach in this paper is reduced obviously and the error is changing with the angular velocity when the SNR is lower and exposure time is only $10 \mathrm{~ms}$. The drift in the output data of MEMS-Gyro has got valid estimates.

\section{Conclusion}

The simulation verifies the effectiveness of the high-performance approach for determining the centroid of star spot which based on MEMS-Gyro's data deep coupling has been proposed in this paper. This approach can help star tracker to maintain high precision when the exposure time is very short, so the update rate of the star image is raised. The MEMS-Gyro can be used to estimate the position of centroid of the spot and then output the attitude when the star image is not update, this further improves the update rate. At the same time, the precision of the MEMS-Gyro has also been raised through the estimate of drift.

It can be seen that the approach can be used to improve the accuracy of determination of position of the spot centroid in a certain extent when the satellite is in the threeaxis stabilized state. In the high angular velocity or time-variable acceleration state, the system is able to maintain a high precision under certain circumstances so that the dynamic performance of star tracker has been raised.

The traditional application of EKF in the attitude determination is to obtain the optimal estimation of the attitude quaternion. In contrast to previous, the EKF used in the approach which presented in this paper has two main functions: setting extraction window and estimating the position of centroid of the spot. So, through this approach, the system can obtain the optimal estimation of the spot centroid when the SNR is lower. By this, the renew rate of star tracker is increased and the dynamic performance is raised at the same time. 
Specially, it also can be seen through the simulation that when the number of star spot captured by detector of star tracker is not enough to determine the attitude, the drift in the output data of MEMS-Gyro also can be estimated effectively. And then the precision of MEMS-Gyro is improved to determine the attitude without star tracker when star tracker cannot output the attitude data.

The novel approach for determining the centroid of star spot which based on MEMSGyro's data deep coupling combines both the advantages of star tracker and MEMS-Gyro, optimizes both the performance of them, and improves both the precision of them. And this method can be applied in robotic vision navigation and other attitude determination fields [25].

\section{Acknowledgment}

The authors thank the financial support from National 863 Plans Projects (2012AA121503) and National Natural Science Foundation of China (60807004).

\section{References}

[1] H. Jia, J. Yang, X. Li et al., "Systematic error analysis and compensation for high accuracy star centroid estimation of star tracker," Science China Technological Sciences, vol. 53, no. 11, pp. 3145-3152, 2010.

[2] G. Rufino and D. Accardo, "Enhancement of the centroiding algorithm for star tracker measure refinement," Acta Astronautica, vol. 53, no. 2, pp. 135-147, 2003.

[3] F. Xing, Y. Dong, and Z. You, "Laboratory calibration of star tracker with brightness independent star identification strategy," Optical Engineering, vol. 45, no. 6, Article ID 063604, 2006.

[4] L. Cao, X. Chen, and T. Sheng, "An algorithm for high precision attitude determination when using low precision sensors," Science China, vol. 55, no. 3, pp. 626-637, 2012.

[5] C. Van der Ha Jozef, "Spin-axis attitude determination and covariances in local sun-earth frame," Journal of Guidance Control and Dynamics, vol. 34, no. 6, pp. 1720-1727, 2011.

[6] B. Alandry, L. Latorre, and F. Mailly, "A fully integrated inertial measurement unit: application to attitude and heading determination," IEEE Sensors Journal, vol. 11, no. 11, pp. 2852-2860, 2011.

[7] W. Xiaojuan and W. Xinlong, "Multiple blur of star image and the restoration under dynamic conditions," Acta Astronautica, vol. 68, no. 11-12, pp. 1903-1913, 2011.

[8] R. Antonello and R. Oboe, "Exploring the potential of MEMS gyroscopes," IEEE Industrial Electronics Magazine, vol. 6, no. 1, pp. 14-24, 2012.

[9] R. Odiander, M. G. Darrin, and J. L. Champion, MEMS and Microstructures Aerospace Application, CRC Press, 2006.

[10] R. J. Terrile, H. Aghazarian, M. I. Ferguson et al., "Evolutionary computation technologies for the automated design of space systems," in Proceedings of the NASA/DoD Conference on Evolvable Hardware (EH'05), pp. 131-138, July 2005.

[11] D. Keymeulen, C. Peay, D. Foor et al., "FPGA platform for MEMS Disc Resonance Gyroscope (DRG) control," in Proceedings of the Micro (MEMS) and Nanotechnologies for Space, Defense, and Security II, March 2008.

[12] T. Brady, S. Buckley, and C. Tillier, "Ground validation of the inertial stellar compass," in Proceedings of the IEEE Aerospace Conference Proceedings, pp. 214-225, March 2004.

[13] L. Blarre, N. Perrimon, and S. Airey, "New multiple head Star Sensor (HYDRA) description and development status: a highly autonomous, accurate and very robust system to pave the way for gyroless very accurate AOCS systems," in Proceedings of the AIAA Guidance, Navigation, and Control Conference, pp. 817-825, August 2005.

[14] N. Chen, F. Xing, and Z. You, "A new approach to determine the controid of star spot for star tracker based on MEMS-Gyro," in Proceedings of the 14th Annual Conference of the Chinese Society of Micro-Nano Technology (CSMNT '12), Hangzhou, China, November 2012. 
[15] Y. Jun, L. Bin, and Z. Tao, "A novel systematic error compensation algorithm based on least squares support vector regression for star sensor image centroid estimation," Sensors, vol. 11, no. 8, pp. 73417363, 2011.

[16] U. Schmidt, "Intelligent error correction method applied on active pixel sensor based star tracker," in Proceedings of the Detectors and Associated Signal Processing II, September 2005.

[17] C. Fan and Z. You, "Highly efficient sigma point filter for spacecraft attitude and rate estimation," Mathematical Problems in Engineering, vol. 2009, Article ID 507370, 2009.

[18] H. Baiqing, C. Lubin, and L. An, "Comment on, 'highly efficient sigma point filter for spacecraft attitude and rate estimation'," Mathematical Problems in Engineering, vol. 2012, Article ID 170391, 5 pages, 2012.

[19] K. Xiong, C. Q. Zhang, and L. D. Liu, "Identification of star sensor low-frequency error parameters," IET Control Theory \& Applications, vol. 6, no. 3, pp. 384-393, 2012.

[20] H.-B. Liu, J.-K. Yang, J.-Q. Wang, J.-C. Tan, and X.-J. Li, “Star spot location estimation using Kalman filter for star tracker," Applied Optics, vol. 50, no. 12, pp. 1735-1744, 2011.

[21] S. Hoday and T. Masayoshi, "Multiple model adaptive estimation of satellite attitude using MEMS Gyros," in Proceedings of the American Control Conference (ACC '11), pp. 3490-3495, San Francisco, Calif, USA, July 2011.

[22] G.-X. Shi, S.-X. Yang, and Z. Su, "Random drift suppression method of MEMS gyro using federated Kalman filter," in Proceedings of the 3rd International Conference on Advanced Computer Control (ICACC '11), pp. 274-277, January 2011.

[23] X. Fei, D. Ying, Y. Zheng, and Z. Qin, "APS star tracker and its attitude estimation," in Proceedings of the 1st International Symposium on Systems and Control in Aerospace and Astronautics, pp. 34-38, Harbin, China, January 2006.

[24] J. Shen, G. Zhang, and X. Wei, "Simulation analysis of dynamic working performance for star trackers," Journal of the Optical Society of America A, vol. 27, no. 12, pp. 2638-2647, 2010.

[25] S. Y. Chen, "Kalman filter for robot vision: a survey," IEEE Transactions on Industrial Electronics, vol. 59, no. 11, pp. 4409-4420, 2011. 


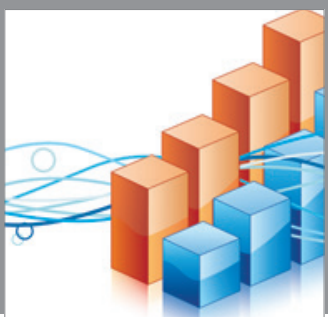

Advances in

Operations Research

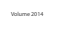

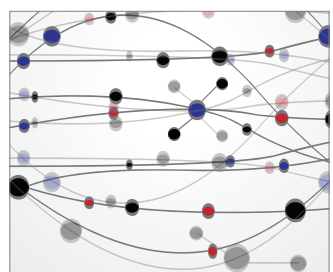

\section{The Scientific} World Journal
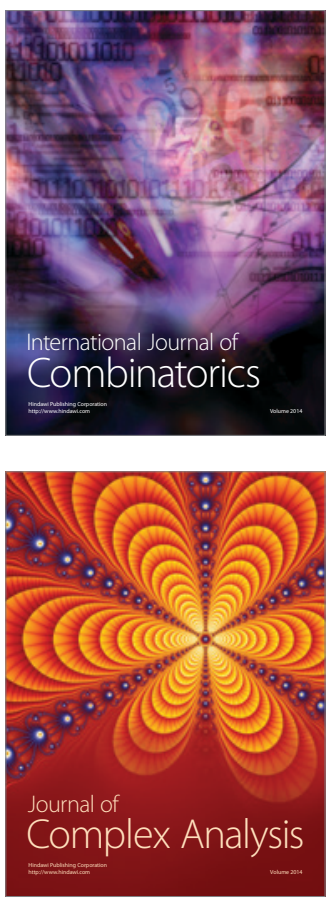

International Journal of

Mathematics and

Mathematical

Sciences
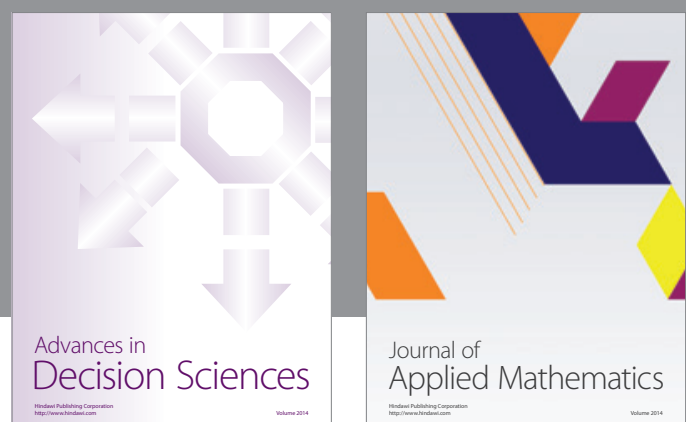

Journal of

Applied Mathematics
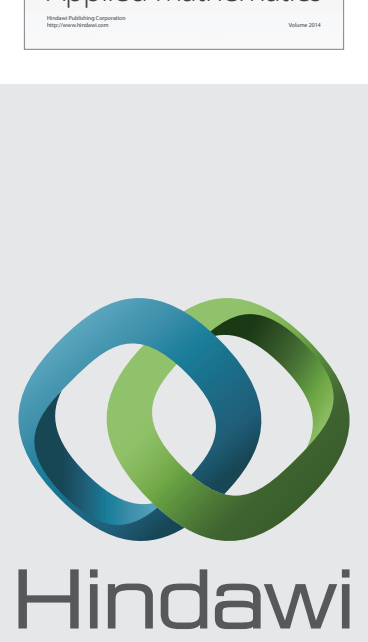

Submit your manuscripts at http://www.hindawi.com
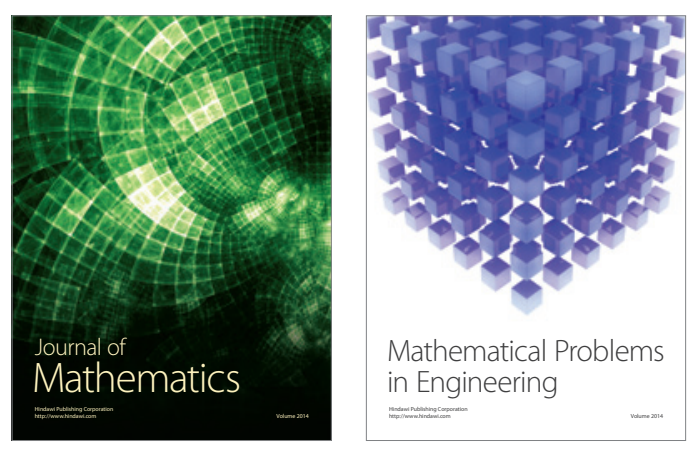

Mathematical Problems in Engineering
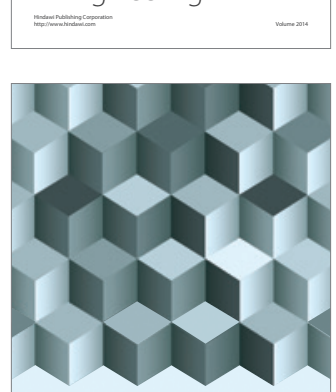

Journal of

Function Spaces
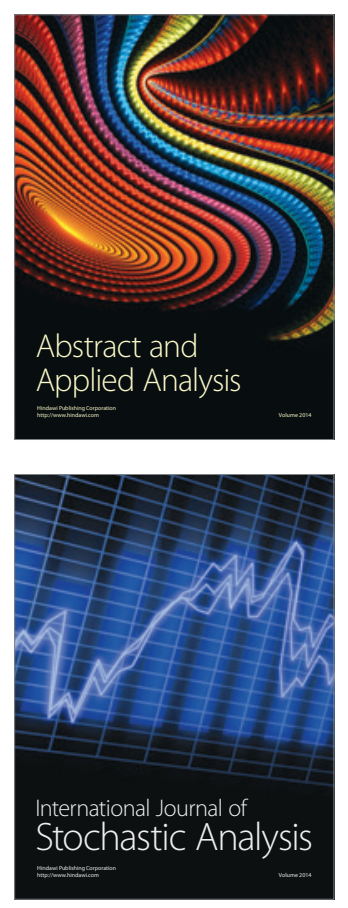

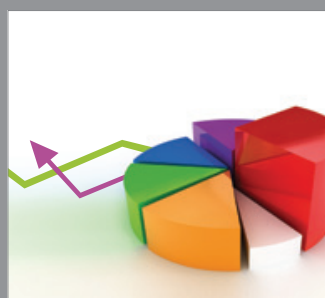

ournal of

Probability and Statistics

Promensencen
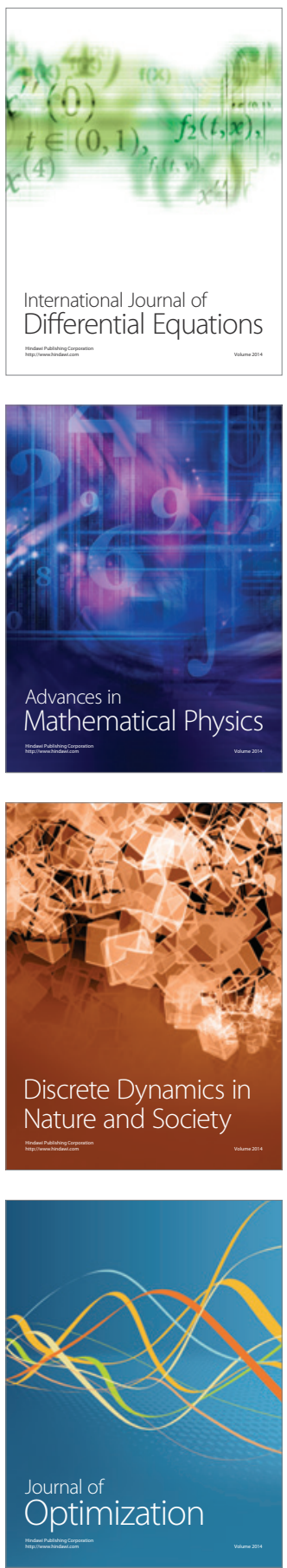